the minimum binge count ( 4 drinks for females, 5 drinks for males). METHODS/STUDY POPULATION: We analyzed data from nontreatment seeking volunteers enrolled in NIAAA screening protocols. The sample included 706 males and 474 females ranging in age from 18 to 91 . Subjects were assigned to one of four groups (Non-Binge, Level 1, Level 2, Level 3) based on the highest binge session reported in their Timeline Followback questionnaire. The criteria for each group were different for males and females based on the current NIAAA definitions of binge drinking. The cutoffs for females were 0-3 drinks for Non-Binge, 4-7 drinks for Level 1, 8-11 drinks for Level 2, and 12+ drinks for Level 3. The male drink cutoffs were $0-4,5-9,10-14$, and $15+$ respectively. We looked at various drinking measures (Timeline Followback, Self-Reported Effects of Alcohol (SRE), Alcohol Use Disorders Identification Test (AUDIT)) and trait measures (UPPS-P Impulsivity Scale, Barratt's Impulsiveness Scale, Buss Perry Aggression Questionnaire) to identify mean differences between groups. RESULTS/ANTICIPATED RESULTS: There were significant differences in drinking patterns between the groups for both males and females. Number of drinking days, average drinks per drinking day, and number of heavy drinking days all increased as binge level increased. There were also significant differences between groups in males for trait measures. Level 2 and Level 3 bingers scored significantly higher on impulsivity and aggression than the Level 1 and Non-Binge groups. Ongoing analyses are examining differences among binge groups on other measures including SRE and AUDIT. Future analyses will explore potential mechanisms underlying the relationships between trait measures and binge drinking using structural equation modeling. DISCUSSION/SIGNIFICANCE OF IMPACT: This study found significant differences between high-intensity drinkers, or "super bingers", and lighter binge and non-binge drinkers. Super bingers showed an overall heavier drinking pattern across measures. The elevated aggression, impulsivity, and overall heavy drinking patterns of super bingers suggest a behavioral profile that makes this group in particular at higher risk for developing alcohol use disorder and related problems. These traits and behaviors may also help identify targets for treatment interventions for alcohol use disorder.

3352

\section{Surgical Adjuvant of Immunomodulatory Gene Circuits for Treatment of Glioblastoma}

Jordan Matthew Spatz ${ }^{1}$, Ming Ru Wu ${ }^{2}$, Karen Weisinger ${ }^{2}$, Tim $\mathrm{Lu}^{2}$ and Manish Aghi, MD, $\mathrm{PhD}^{1}$

${ }^{1}$ University Of California, San Francisco and ${ }^{2}$ M.I.T.

OBJECTIVES/SPECIFIC AIMS: Glioblastoma (GBM) is a brain cancer with a devastatingly short overall survival of under two years. The poor prognosis of GBM is largely due to cell invasion and maintenance of cancer initiating cells that evade the brain's innate and adaptive immune responses which enables escape from surgical resection and drives inevitable recurrence. While targeting the brain's immune microenvironment has long been proposed as a strategy for treating GBM, translational progress has been slow, underscoring the need to investigate the brain's immune microenvironment for therapeutic avenues. METHODS/STUDY POPULATION: Recent advancements in tunable synthetic immunomodulatory gene circuits targeting metastatic cancers has demonstrated the novel ability to use engineering principles to induce infiltrative cancer cells to express combinatorial immunomodulatory outputs that enable T-cell killing4. Our central hypothesis is: we will be able to significantly improve survival with a lasting immunemediated control of GBM by using synthetic immunomodulatory gene circuits driving GBM cells to express a local combination of immunomodulatory proteins: human IL15, a surface T-cell engager, PD-L1-CD3 bispecific antibody, and the protein, LIGHT (TNFRSF14). Importantly, the co-expression of LIGHT and antiPD-L1 therapies was recently shown to rescue PD-L1 checkpoint blockage in the preclinical models of brain tumors and significant enhance survival outcomes highlighting the benefits of novel combinations of immunomodulatory proteins for treatment of GBM. To identify genes whose expression is dramatically upregulated in GBM compared to normal human brain cells, a pooled of six thousand lentiviral oncogene promoters that drive expression of a redfluorescent protein has been infected into three human GBM cell lines. RESULTS/ANTICIPATED RESULTS: We have successfully infected our GBM cells and are preparing samples for next generation DNA sequencing to determine highly active promoters in GBM that are not expressed in multiple normal brain cells types, astrocytes and neurons. These chosen promoters will then be used to drive an AND gate logic gene circuit immunotherapy outputs which is currently under development for both in-vitro and in-vivo experiments. DISCUSSION/SIGNIFICANCE OF IMPACT: We anticipate that local expression of multiple immune effectors proteins will significantly enhance tumor control and survival in both synergistic murine and human-murine xenograft pre-clinical models of GBM. Ultimately, our goal is to rapidly translate this technology advance into the clinical trial for adult GBM patients.

\section{TARGETING DIABETES PREVENTION PROGRAMS: INDIVIDUAL RISK-BASED HEALTH ECONOMIC ANALYSIS}

Natalia Olchanski ${ }^{1}$, David van Klaveren, Joshua T Cohen, John B Wong, Robin Ruthazer and David M Kent

${ }^{1}$ Center for the Evaluation of Value and Risk in Health, Tufts Medical Center

OBJECTIVES/SPECIFIC AIMS: Objective: Approximately 86 million people in the US have prediabetes, but only a fraction of them receive proven effective therapies to prevent diabetes. Further, the effectiveness of these therapies varies with individual risk of progression to diabetes. We estimated the value of targeting those individuals at highest diabetes risk for treatment, compared to treating all individuals meeting inclusion criteria for the Diabetes Prevention Program (DPP). METHODS/STUDY POPULATION: METHODS: Using a micro-simulation model, we estimated total lifetime costs and qualityadjusted life expectancy (QALE) for individuals receiving: (1) lifestyle intervention involving an intensive program focused on healthy diet and exercise, (2) metformin administration, or (3) no intervention. The model combines several components. First a Cox proportional hazards model predicted onset of diabetes from baseline characteristics for each pre-diabetic individual and yielded a probability distribution for each alternative. We derived this risk model from the Diabetes Prevention Program (DPP) clinical trial data and the follow-up study DPP-OS. The Michigan Diabetes Research Center Model for Diabetes then estimated costs and outcomes for individuals after diabetes diagnosis using standard of care diabetes treatment. Based on individual costs and QALE, we evaluated $\mathrm{NMB}$ of the two interventions at population and individual levels, 